\title{
Predictability of the resonance frequency analysis in the survival of dental implants placed in the anterior non-atrophied edentulous mandible
}

\author{
Raúl González-García ${ }^{1}$, Florencio Monje ${ }^{2}$, Carlos Moreno-García ${ }^{2}$
}

${ }^{1} \mathrm{MD}$, Staff Surgeon. Department of Oral and Maxillofacial Surgery, University Hospital Infanta Cristina, Badajoz, Spain
${ }^{2} \mathrm{MD}$, PhD. Oral and Maxillofacial Surgeon, CICOM, Centro de Implantología y Cirugía Oral y Maxilofacial, Badajoz, Spain

Correspondence:

Calle Los Yébenes 35, 8C

28047, Madrid, Spain

raulmaxilo@gmail.com

\author{
González-García R, Monje F, Moreno-García C. Predictability of the re- \\ sonance frequency analysis in the survival of dental implants placed in \\ the anterior non-atrophied edentulous mandible. Med Oral Patol Oral Cir \\ Bucal. 2011 Aug 1;16 (5):e664-9. \\ http://www.medicinaoral.com/medoralfree01/v16i5/medoralv16i5p664.pdf

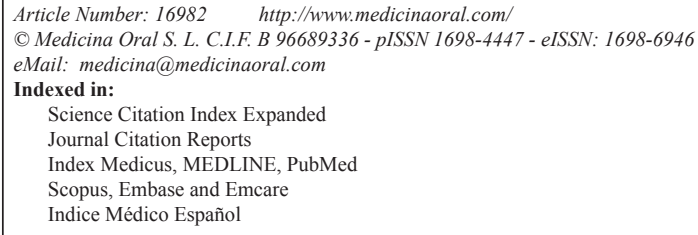

\begin{abstract}
Background: Dental primary implant stability is considered essential in the success of the osseointegration process. The recent advent of the resonance frequency analysis (RFA) seems to effectively measure primary implant stability, although its relationship with implant survival has to be further established.

Patients and Methods: Seventy patients with complete mandibular edentulism underwent dental implant rehabilitation by means of the placement of 68 dental implants within the interforaminal region and subsequent placement of an overdenture. Primary implant stability was measured by means of RFA and it was expressed in terms of implant stability quotient (ISQ) on the day of the implant insertion and at the time of the healing abutment placement in a conventional implant two-stage surgical procedure.

Results: Overall implant survival rate was $97.1 \%$ at the end of the follow-up period. The mean ISQ value for 3.75 and $4.25 \mathrm{~mm}$ diameter implants was $78.4 \pm 5.46$ and $80.83 \pm 5.35$ respectively, at the time of the implant placement; and $76.68 \pm 4.34$ and $78.22 \pm 6.87$ respectively, at the second surgical stage. No statistical differences were observed in relation to changes in mean ISQ value along the healing process.

Conclusions: No statistical differences in terms of primary and secondary implant stability measured by RFA exists between $3.75 \mathrm{~mm}$ and $4.25 \mathrm{~mm}$ diameter implants in the conventional implant two-stage surgical procedure in patients with non-atrophied edentulous mandible being restored with an overdenture. Furthermore, no statistical association between RFA and the implant insertion torque was observed for endosseous dental implant placement at the first surgical stage.
\end{abstract}

Key words: Resonance frequency analysis, endosseous dental implants, anterior mandible, implant survival, primary and secondary implant stability. 


\section{Introduction}

The use of endosseous dental implants is a very popular alternative for oral rehabilitation, since the introduction of the osseointegration concept by Brånemark in 1985. Successful result of the implant insertion procedure depends on factors related to the patient and also to the procedure, including general health status, bio-compatibility of the implanted material, micro- and macroscopic nature of the implant surface, surgical procedure, and quality and quantity of local bone.

It is considered that primary implant stability plays a major role in the success of osseointegration (1). It depends on the quality and quantity of bone, the geometry of the implant and the technique for preparation of the implant site (2). Volume and quality of bone are important factors for determining the surgical procedure and the choice of the implant, and are related to the success of implant surgery (3). The resonance frequency analysis (RFA) technique measures implant stability as a function of the complex bone-implant rigidity. Resonance frequency (measured in $\mathrm{Hz}$ ) is established by the value of the implant stability quotient (ISQ). Implant stability is typically measured between 45 and 85 ISQ, and is mostly determined by bone density at the implant site.

RFA has been previously reported to be effective in the detection of implant failure and in the checking of the healing process $(4,5)$. Furthermore, it has been reported the importance of cortical bone in the determination of primary stability, with a positive correlation between RFA and the height of cortical bone in experimental studies with animals (6). Correlation in the patient alive has been studied less extensively.

Based upon these previous considerations, the present study analyzes the correlation between primary and secondary stability of endosseous dental implants placed over the anterior mandible of edentulous patients without bone atrophy, and also analyzes implant survival. It also analyzes the influence of the implant diameter in the determination of primary and secondary stability, and its correlation with RFA values.

\section{Materials and Methods}

Sixty-eight endosseous dental implants MG-Osseous (Mozo-Grau S.L, Valladolid, Spain) were used. They were self-screw type Brånemark implants with external hexagon and RBM surface. The object of the study was defined as the number of inserted implants. Two implant diameters 3.75 and $4.25 \mathrm{~mm}$ were used in relation to the width of the anterior alveolar crest. The timetable of the study was: 1) inclusion of patients undergoing implant placement due to the absence of teeth in the non-atrophied anterior mandible; 2) insertion of endosseous dental implants in the anterior mandible for an overdenture protocol; 3) measurement of ISQ values by RFA in the first surgical procedure and placement of tapping screws; 4) removal of tapping screws during the second surgical time, measurement of ISQ values and placement of healing abutments (Fig. 1. A,B,C); 5) evaluation of implant survival and statistical analysis of results.

During the first surgical stage, drilling sequence was performed according to manufacturer's recommendations: 1) initial drill; 2) $2.0 \mathrm{~mm}$ diameter drill; 3) $3.0 \mathrm{~mm}$ diameter drill; 4) $3.3 \mathrm{~mm}$ diameter drill (last drill for $3.75 \mathrm{~mm}$ diameter implants); 5) $3.8 \mathrm{~mm}$ diameter drill (last drill for $4.25 \mathrm{~mm}$ diameter implants); and 6) drill

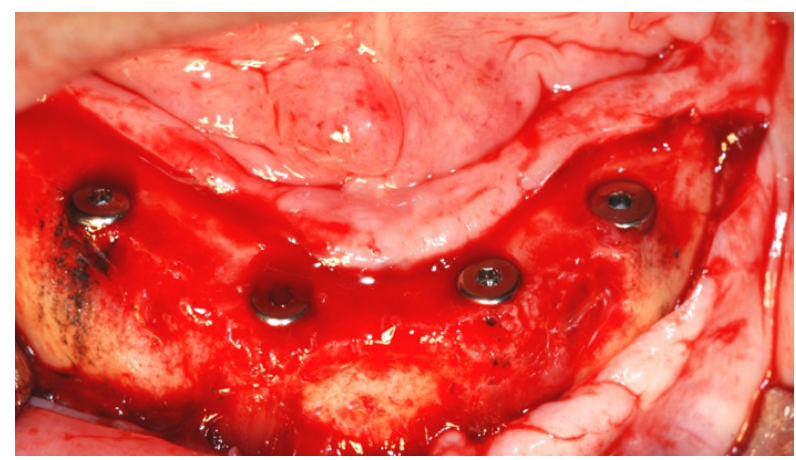

Fig. 1A. Implant insertion at the first surgical stage.

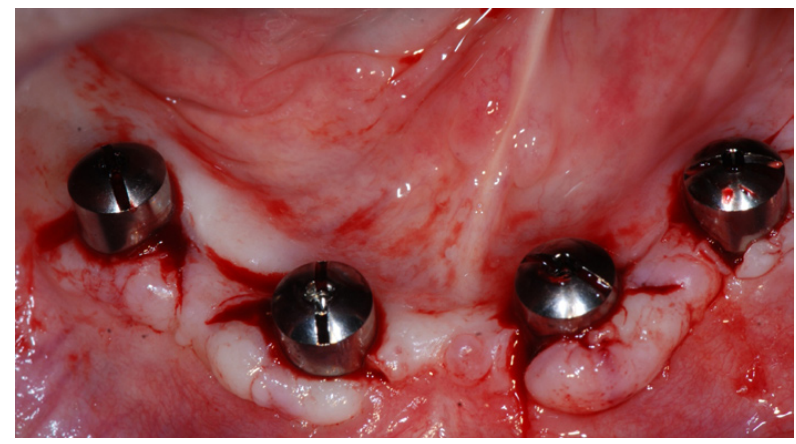

Fig. 1B. Placement of healing abutments at the second surgical stage.

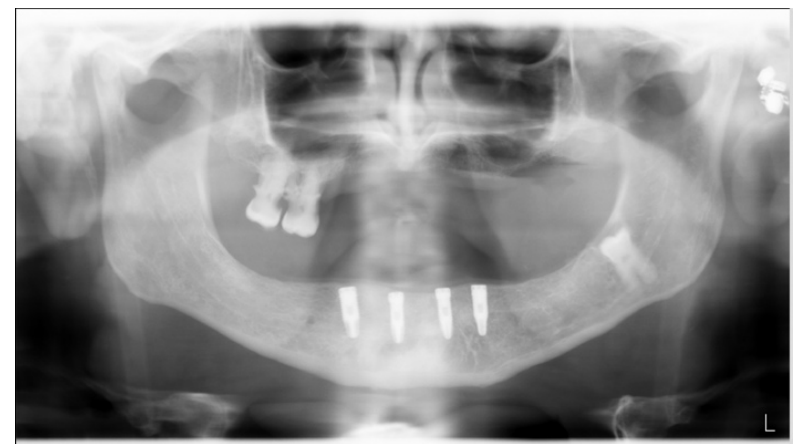

Fig. 1C. Panoramic radiography showing implant position at the immediate post-operative time following the first surgical stage. 
for smoothing cortical bone (for both implant diameters). Drilling was applied at $1000 \mathrm{rpm}$, under abundant continuous internal irrigation. Implant insertion torques were 35, 40 and $45 \mathrm{Ncm}$, in different proportions.

Patients' inclusion criteria included: 1) complete mandibular edentulism, with adequate preservation of the alveolar mandibular height and width; 2) placement of endosseous dental implants for overdenture fixation in non-atrophied anterior mandible; 3) absence of systemic pathology; 4) absence of smoking habit. Implants were placed following the conventional technique in two steps, with insertion during the first surgical stage and placement of the healing abutments during the second surgical stage, at least 3 months later. RFA was measured by the Ostell ${ }^{\mathrm{TM}}$ Mentor (Integrations Diagnostics AB, Savedalen, Sweden) instrument (7) and quantification was performed by the ISQ value. Measurements were taken twice in the anterior-posterior and lateralmedial vectors during first and second surgical stages. Prosthetic rehabilitation consisted on the placement of a mandibular overdenture option SD-4, with implants in positions $\mathrm{A}, \mathrm{B}, \mathrm{D}$ and $\mathrm{E}$, rigidly fixed with a $10-\mathrm{mm}$ distal cantilevered bar.

Statistical package SPSS 13.0 (SPSS Inc., Chicago, IL, US) was used to analyze data. Descriptive statistical analysis for continuous and categorical variables was performed. Student's t test for paired samples was used to compare mean ISQ values between first and second surgical stage. In an attempt to analyze the influence of implant diameter in primary and secondary implant stability measured by RFA, $3.75 \mathrm{~mm}$ diameter implants were compared with $4.25 \mathrm{~mm}$ diameter implants by the Student's t test for unpaired samples. P value $\leq .05$ was considered statistically significant.

\section{Results}

Mean age of the whole series was $61.03 \pm 9.71$ years. Sixty-eight endosseous dental implants were placed, with an equal distribution among genders $(50 \%$ placed in male patients and $50 \%$ placed in female patients). Fifty $3.75 \mathrm{~mm}$ diameter implants $(73.5 \%)$ and eighteen 4.25 mm diameter implants $(26.5 \%)$ were inserted. Overall implant success rate was $97.1 \%$ at the end of the followup period, with failure of 2 implants, 3.75 and $4.25 \mathrm{~mm}$ diameter each. In these cases, a decrease upper than 10 ISQ points was observed in RFA between first and second surgical stages for both implant sizes.

Mean insertion torque for the whole series was $37.65 \pm$ $3.39 \mathrm{Ncm}$, with $39(57.4 \%)$ implants inserted at $35 \mathrm{Ncm}$, $22(32.4 \%)$ at $40 \mathrm{Ncm}$, and $7(10.3 \%)$ at $45 \mathrm{Ncm}$. Mean ISQ value obtained by the RFA during implant insertion stage was $79.04 \pm 5.50$, while mean ISQ value at the second surgical stage for healing abutments connection was $77.09 \pm 5.12$. Mean time between both surgical stages was $114.51 \pm 24.71$ days (range: 90-191 days). In relation to bone features according to the Lekholm \& Zarb classification, bone type I was observed in $78.6 \%$ of the inserted implants, bone type II in $14.3 \%$, bone type III in 5.4\%, and bone type IV in $1.8 \%$. Considering scores 1 to 4 for bone types I to IV respectively, mean value of the series in relation to bone type was $1.30 \pm$ 0.65 .

In the analytic study, significant statistical differences in relation to AFR mean values between the first and second surgical stage were encountered $(p=0.031)$. Mean ISQ value for $3.75 \mathrm{~mm}$ diameter implants at the first surgical stage was $78.40 \pm 5.46$, and decreased to 76.68 \pm 4.34 at the second surgical stage (measured $109.74 \pm$ 20.92 days post-surgery). Mean ISQ value for $4.25 \mathrm{~mm}$ diameter implants at the first surgical stage was $80.33 \pm$ 5.35 , and decreased to $78.22 \pm 6.87$ at the second surgical stage (measured $127.78 \pm 29.83$ days post-surgery). Following the application of the Student's t test for unpaired data, no significant differences were observed between implant diameters $3.75 \mathrm{~mm}$ and $4.25 \mathrm{~mm}$, in relation to primary and secondary implant stability measured by RFA ( $p=0.11$ and $p=0.38$, respectively). No statistically significant association was either observed between insertion torques and ISQ values during implant insertion at first and second surgical stages.

No statistically significant association was either observed between sex and age and ISQ values at the first and second surgical stages. Due to the scarce number of failed implants, and the fact that these implants were of both diameters, analyses of the influence of the ISQ value in the implant success rate was inapplicable, and no parameter was compared with such variable. Although in the present study, both failed implants showed a decrease in ISQ values of 10 and 12 points respectively, a difference equal or major than 10 points between final ISQ value and initial ISQ value did not showed a statistically significant association with implant success rate. Furthermore, 13 (19.11\%) endosseous dental implants showed differences equal or major than 10 points between final ISQ value and initial ISQ value, without an observed failure of the osseointegration process in 11 of $13(84.61 \%)$ implants.

\section{Discussion}

The presence of significant higher ISQ values in the anterior site of the mandible than in the posterior site has been previously reported in the literature (8). The width of the implant has also been reported to be an important factor in relation to supported prosthetic loads. As far as the implant is wider, the more decrease of loads is observed due to an increase of the implant surface, as well as the implant length can be decreased. In fact, width has been reported to be more relevant than length, as highest loads concentrate in the coronal zone (7). The fact that each $0.5 \mathrm{~mm}$ of implant width increase corres- 
ponds with an increase of $10 \%$ to $15 \%$ of the implant surface, lead us to study the influence of two implant diameters: 3.75 and $4.25 \mathrm{~mm}$ (differing each other 0.5 $\mathrm{mm}$ exactly) in the implant stability, measured primarily and secondarily by RFA. In the present study, no significant statistical differences in terms of primary and secondary implant stability were observed between both diameters, although bone type D4 in the Misch classification (7) has been reported to more commonly require wider implants, in contrast to those required by bone type D1 and D2. This disagreement may be attributable to the preponderance of implants over cortical bone in the present series, corresponding to subtypes 1 and 2 of the Lekholm \& Zarb classification.

In a study about 48 inserted implants in cadaver by Nkenke et al. (9), RFA and histo-morphometric study was performed for every implant. Several items were analyzed: 1) bone-implant contact; 2) trabecular pattern factor (TBPf): it measures the degree of inter-trabecular connectivity, which increases as TBPf decreases; 3) trabecular bone density (bone volume/total volume, $\mathrm{BV} / \mathrm{TV}$ ); and 4) cortical bone height trespassed by the implant. Mean values by RFA (maxilla $6130.4 \pm 363.2$ $\mathrm{Hz}$, mandible $6424.5 \pm 236.2 \mathrm{~Hz}$ ) did not correlate with bone mineral density (maxilla $259.2 \pm 124.8 \mathrm{mg} / \mathrm{cm}^{3}$, mandible $349.3 \pm 113.3 \mathrm{mg} / \mathrm{cm}^{3}$ ), with BV/TV (maxilla $19.7 \pm 8.8 \%$, mandible $34.3 \pm 6.0 \%$ ), and neither with TNPf (maxilla $2.39 \pm 1.46 \mathrm{~mm}-1$, mandible $-0.84 \pm$ $3.27 \mathrm{~mm}-1)$. However, the authors found a correlation between RFA and bone-implant contact (maxilla 12.6 $\pm 6.0 \%$, mandible $35.1 \pm 5.1 \%$ ) and between RFA and cortical bone height trespassed by implants (maxilla $2.1 \pm 0.7 \mathrm{~mm}$, mandible $5.1 \pm 3.7 \mathrm{~mm}$ ). They concluded that non-invasive measurement of implant stability by RFA had to be improved to allow a more comprehensive prediction of implant site features. In the present series, inclusion of patients without mandibular atrophy is evident by the presence of bone with a mean value of $1.30 \pm$ 0.65 in the Lekholm \& Zarb classification, and the fact that $78.6 \%$ of the implants were inserted in bone type 1 , $14.3 \%$ in bone type 2 , while only $7.2 \%$ in bone types 3 and 4 . These results differ slightly from those observed by Misch (7), who reported a distribution of $6 \%, 66 \%$, $25 \%$ and $3 \%$ for bone densities D1, D2, D3, and D4, respectively, in the anterior mandible. The presence of a high rate of patients with abundant cortical bone in our series may explain the relative high mean values in the RFA measured in the first and second surgical stages: 79.04 and 77.09 , respectively. In fact, we wanted to test the reported implants only in a selected group of patients with mandibular edentulism without atrophy in the anterior site. Otherwise, this issue can be determinant in the observed high implant success rate.

Mean ISQ value at the implant insertion was higher than that for global series (10), which indicates the pre- dictability in terms of primary stability placed in the non-atrophied anterior mandible. Huwiler et al. (11), in a series of 17 implants placed in the patient alive, observed a normal ISQ range between 55 and 74 at the time of implant insertion. They also observed an increase in ISQ values during the week following implant insertion, followed by a decrease during weeks 2 to 4, and a posterior increase to ISQ values obtained during the insertion phase, or even higher. Loss of implant stability occurring one week after implant insertion can be attributable to loss of mechanical stability identified during the early healing period, which has been previously described by Abrahamsson et al. (12). Otherwise, the osseointegration phase by contact osseogenesis, previously documented in animal studies after 2-4 wee$\mathrm{ks}$, is represented by the slight increase of ISQ values observed during the late phase of the healing process, from the fourth week. Based on the failure of an implant, which lost stability after 3 weeks with an important decrease of 20 ISQ units, from 68 to 45 in a week, authors conclude that RFA is a specific but not sensitive biomechanic test of implant stability, without predictive value for implant failure, as far as the observed low ISQ value was found once the implant had already lost stability, and also the ISQ value of 45 was marginal and no significant difference was observed with respect to ISQ range of the rest of the series. Interestingly, these results are quite similar to those obtained in the present study, as we could not demonstrate a statistically significant association between loss of ISQ units and failure of implant osseointegration. In fact, an $84.61 \%$ of the implants loosing 10 or more ISQ points showed successful osseointegration despite decrease of implant stability values, in contrast to $15.39 \%$ that did not osseointegrate. Because of the design of the study and due to the fact that it was performed in patients alive, it was impossible to serially evaluate the evolution of post-operative ISQ values along the weeks that followed implant insertion, and thus final ISQ measurement was performed at the second surgical stage during the placement of healing abutments. Abrahamsson et al. (12), in animal studies, report a gradually increase in ISQ values after an initial decrease, from the $4^{\text {th }}$ week following implant insertion. The evaluation of mean RFA at the $16^{\text {th }}$ week (range 12 to 27 weeks) performed in our study showed a decrease of mean ISQ value up to 1.95 units, which was significantly different, although it did not have any clinical influence, as implant osseointegration did not change. Correlation of ISQ values decrease at the $16^{\text {th }}$ week with bone morphogenesis at the bone-implant interface must be analyzed further in future studies, although high initial ISQ values and the presence of bone type 1 and 2 in a large number of patients could explain results observed in the present study.

RFA has showed an important sensibility in monitoring 
implant stability changes. Friberg et al. (1) showed an increase of implant stability for implants in the upper maxilla from insertion to the second surgical stage at the placement of healing abutments, and further increase one year later to the prosthetic load. In a clinical study with bone graft in the maxilla, Rasmusson et al. (13) reported an increase in ISQ values along the time, and recommended the use of small diameter drills for implant insertion, in order to generate high compression forces that allow higher stability, at least initially. Similarly, Glauser et al. (14), in a study using RFA in 81 dental implants for a year, observed a continuous decrease of implant stability that finally failed. They concluded that low ISQ values after 1 to 2 months seem to indicate a higher risk for a future implant failure. This information can be useful to avoid a potential implant failure, unloading implants with a decreasing implant stability diagnosed by RFA. In our series, implant stability in the moment of the healing abutment connection was slightly lower, although final ISQ values were in the rank of implant success previously reported in the literature. Kessler-Liechti et al. (15), in a study over 205 implants, observed a mean ISQ of 64.5 for interforaminal implants that were asymptomatic at any time of the follow-up. These authors, however, did not observe any significant differences in terms of ISQ values at different post-surgical times.

Among three methods for the evaluation of implant stability (RFA, Periotest and insertion torque), only RFA has been correlated to bone-implant contact in experimental studies with animals $(6,16)$. However, in the study over maxilla and mandible of human cadaver this correlation was less pronounced, probably because properties of human mandible and maxilla change rapidly in a short period of time. In relation to these results, we did not find a statistically significant association between RFA and insertion torque at the non-atrophied edentulous interforaminal mandible.

Two aspects not evaluated in the present study seem to play a major role in the determination of implant stability: bone-implant contact surface and height of alveolar cortical bone. In fact, previous studies have correlated RFA with height of the alveolar cortical bone. Also, recent bibliography in relation to implant failure evaluate peri-implantitis as the loss of vertical bone around an implant more than $4 \mathrm{~mm}$ during the first year, and 1.5 $\mathrm{mm}$ each following year (16-18). Design of prospective studies that acutely evaluate these parameters may provide important information about the ability to predict implant survival in relation to primary and secondary stability, influenced by bone density, height of alveolar cortical bone and bone-implant contact surface.

\section{Conclusions}

In the present series of edentulous patients with nonatrophied mandible restored by an overdenture, the resonance frequency analysis at the $16^{\text {th }}$ week from the endosseous dental implant insertion showed a significant decrease of 2 ISQ units, although no significant differences in terms of primary and secondary implant stability among 3.75 and $4.25 \mathrm{~mm}$ diameter implants were encountered in the traditional two-step implant rehabilitation procedure. Otherwise, no statistical association between the resonance frequency analysis and the implant insertion torque value at the first surgical stage was encountered.

\section{References}

References with links to Crossref - DOI

1. Friberg B, Jemt T, Lekholm U. Early failures in 4,641 consecutively placed Brånemark dental implants: a study from stage 1 surgery to the connection of completed prostheses. Int J Oral Maxillofac Implants. 1991;6:142-6.

2. Sennerby L, Roos J. Surgical determinants of clinical success of osseointegrated oral implants: a review of the literature. Int J Prosthodont. 1998;11:408-20.

3. Ekfeldt A, Christiansson U, Eriksson T, Lindén U, Lundqvist S, Rundcrantz $\mathrm{T}$, et al. A retrospective analysis of factors associated with multiple implant failures in maxillae. Clin Oral Implants Res. 2001;12:462-7.

4. Sennerby L, Persson LG, Berglundh T, Wennerberg A, Lindhe J. Implant stability during initiation and resolution of experimental periimplantitis: an experimental study in the dog. Clin Implant Dent Relat Res. 2005;7:136-40.

5. Brouwers JE, Lobbezoo F, Visscher CM, Wismeijer D, Naeije M. Reliability and validity of the instrumental assessment of implant stability in dry human mandibles. J Oral Rehabil. 2009;36:279-83.

6. Meredith N, Book K, Friberg B, Jemt T, Sennerby L. Resonance frequency measurements of implant stability in vivo. A cross-sectional and longitudinal study of resonance frequency measurements on implants in the edentulous and partially dentate maxilla. Clin Oral Implants Res. 1997;8:226-33.

7. Capek L, Simunek A, Slezak R, Dzan L. Influence of the orientation of the Osstell transducer during measurement of dental implant stability using resonance frequency analysis: a numerical approach. Med Eng Phys. 2009;31:764-9.

8. Boronat López A, Balaguer Martínez J, Lamas Pelayo J, Carrillo García C, Peñarrocha Diago M. Resonance frequency analysis of dental implant stability during the healing period. Med Oral Patol Oral Cir Bucal. 2008;13:E244-7.

9. Nkenke E, Hahn M, Lell M, Wiltfang J, Schultze-Mosgau S, Stech $\mathrm{B}$, et al. Anatomic site evaluation of the zygomatic bone for dental implant placement. Clin Oral Implants Res. 2003;14:72-9.

10. Boronat-López A, Peñarrocha-Diago M, Martínez-Cortissoz O, Mínguez-Martínez I. Resonance frequency analysis after the placement of 133 dental implants. Med Oral Patol Oral Cir Bucal. 2006;11:E272-6.

11. Huwiler MA, Pjetursson BE, Bosshardt DD, Salvi GE, Lang NP. Resonance frequency analysis in relation to jawbone characteristics and during early healing of implant installation. Clin Oral Implants Res. 2007;18:275-80.

12. Abrahamsson I, Berglundh T, Linder E, Lang NP, Lindhe J. Early bone formation adjacent to rough and turned endosseous implant surfaces. An experimental study in the dog. Clin Oral Implants Res. 2004;15:381-92.

13. Rasmusson L, Meredith N, Cho IH, Sennerby L. The influence of simultaneous versus delayed placement on the stability of titanium implants in onlay bone grafts. A histologic and biomechanic study in the rabbit. Int J Oral Maxillofac Surg. 1999;28:224-31. 
14. Glauser R, Sennerby L, Meredith N, Rée A, Lundgren A, Gottlow $\mathrm{J}$, et al. Resonance frequency analysis of implants subjected to immediate or early functional occlusal loading. Successful vs. failing implants. Clin Oral Implants Res. 2004;15:428-34.

15. Kessler-Liechti G, Zix J, Mericske-Stern R. Stability measurements of 1-stage implants in the edentulous mandible by means of resonance frequency analysis. Int J Oral Maxillofac Implants. 2008;23:353-8.

16. Huang HM, Lee SY, Yeh CY, Lin CT. Resonance frequency assessment of dental implant stability with various bone qualities: a numerical approach. Clin Oral Implants Res. 2002;13:65-74.

17. Ko CC, Douglas WH, DeLong R, Rohrer MD, Swift JQ, Hodges JS, et al. Effects of implant healing time on crestal bone loss of a controlled-load dental implant. J Dent Res. 2003;82:585-91.

18. Huang HM, Tsai CM, Chang CC, Lin CT, Lee SY. Evaluation of loading conditions on fatigue-failed implants by fracture surface analysis. Int J Oral Maxillofac Implants. 2005;20:854-9. 